\title{
COVID-19 inpatients with gastrointestinal onset: sex and care needs' differences in the district of Ferrara, Italy
}

Salvatore Greco ${ }^{1 \dagger}$, Nicolò Fabbri' ${ }^{2 \dagger}$, Alessandro Bella ${ }^{1}$, Beatrice Bonsi ${ }^{1}$, Stefano Parini ${ }^{3}$, Cindy Rocchi ${ }^{3}$, Sara Giaccari ${ }^{3}$, Manuel Gavioli ${ }^{3}$, Angelina Passaro $1,4,5^{*} \mathbb{D}$ and Carlo V. Feo ${ }^{2}$

\begin{abstract}
Background: COVID-19 is characterized by interstitial pneumonia, but a presentation of the disease with digestive symptoms only may occur.

This work was aimed at evaluating: (1) the prevalence of presentation with digestive symptoms only in our cohort of COVID-19 inpatients; (2) differences between patients with and without gastrointestinal onset; (3) differences among males and females with gastrointestinal presentation; (4) outcomes of the groups of subjects with and without gastrointestinal onset.

Method: We retrospectively divided the patients hospitalized with COVID-19 into two groups: (1) the one with digestive symptoms (DSG) and (2) the other without digestive symptoms (NDSG). We compared the subjects of DSG with those of NDSG and males with females in the DSG group only, in terms of demographics (age, sex), inflammation and organ damage indexes, length of stay, in-hospital and 100-day mortality.

Results: The prevalence of gastrointestinal symptoms at presentation was $12.5 \%$. The DSG group showed a prevalence of females, and these tended to a shorter hospital stay; DSG patients were younger and with a higher load of comorbidities, but no differences concerning inflammation and organ damage indexes, need for intensification of care, in-hospital and 100-day mortality were detected. Among DSG patients, males were younger than females, more comorbid, with higher serum CRP and showed a longer length of hospital stay. Survival functions of DSG patients, in general, are more favourable than those of NDSG if adjusted for sex, age and comorbidities.
\end{abstract}

Conclusions: (1) The prevalence of gastrointestinal presentation among hospitalized COVID-19 patients was 12.5\%; (2) DSG patients were on average younger, more comorbid and with a prevalence of females, with a shorter hospital stay; (3) in the DSG group, males had a higher Charlson Comorbidity Score and needed a longer hospital stay; (4) DSG subjects seem to survive longer than those of the NDSG group.

Keywords: SARS-CoV-2, Coronavirus infection, COVID-19, Digestive symptoms, Gastrointestinal onset

*Correspondence: angelina.passaro@unife.it

†Salvatore Greco and Nicolò Fabbri contributed equally to the article

${ }^{5}$ Department of Translational Medicine and Medical Department,

University of Ferrara, University Hospital of Ferrara Arcispedale Sant'Anna,

Via Aldo Moro, 8, 44124 Ferrara, Emilia Romagna, Italy

Full list of author information is available at the end of the article

\section{Background}

Coronaviruses are a group of related RNA-viruses that cause diseases in mammals and birds and they are distributed all over the world; in the late 2019, the third member of this family after SARS-CoV-1(Severe Acute Respiratory Syndrome CoronaVirus 1) and MERS$\mathrm{CoV}$ (Middle East Respiratory Syndrome CoronaVirus) 
appeared for the first time, causing two cases of interstitial pneumonia [1].

SARS-CoV-2 infection can manifest itself in many ways and the set of symptoms (COVID-19, COronaVIrus Disease 2019) ranges from influenza-like symptoms (in $80 \%$ of cases) to a severe form of pneumonia (dyspnea or tachypnea with a $\mathrm{PaO} 2 / \mathrm{FiO} 2$ ratio $<300$ at the blood gas assay and a pulmonary infiltration $>50 \%$ ). Up to $5 \%$ of patients develop rapidly an Acute Respiratory Distress Syndrome (ARDS), with or without acute cardiac damage, related to an underlying systemic hyperinflammation $[2,3]$ that can hesitate in systemic shock (cytokine storm), multiorgan failure, and death.

Fever is the most frequent sign: it may be absent in up to $50 \%$ of patients at the first physical examination, but it seems to be present in more than $90 \%$ of cases during hospitalization; cough is present in $67 \%$ of cases. Atypical symptoms of systemic involvement can be noticed, such as arthralgia, myalgia, asthenia, headache, upper respiratory tract symptoms, and gastrointestinal symptoms (nausea, vomiting, and diarrhea up to $5 \%$ of cases in some experiences, especially during the first periods of pandemic; generalized abdominal pain, anorexia and taste changes were also reported as possible digestive symptoms following SARS-CoV-2 infection) $[4,5]$.

Further data confirmed that approximately $50 \%$ of patients with COVID-19 have detectable viral RNA in the stool [6-8] and the prevalence of gastrointestinal symptoms can be up to $50 \%$. Digestive symptoms were also reported during the epidemics by SARS-CoV-1 and MERS-CoV [9].

As of May 2021, almost 170 million cases of COVID19 confirmed cases and more than 3.5 million deaths in more than 200 countries and territories were reported (source https://www.worldometers.info/coronavirus/).

The pathophysiology of digestive symptoms associated with COVID-19 remains partially unexplained. It seems that they likely occur because the virus enters the target cells through angiotensin-converting enzyme 2 (ACE2) [10], a receptor found in both the upper and lower gastrointestinal tract where it is expressed at nearly 100 -fold higher levels than in respiratory organs $[11,12]$.

Most studies focused on the respiratory involvement of SARS-CoV-2 infection and the majority of patients with a severe form of COVID-19 faced respiratory symptoms only, without digestive symptomatology [13]. It is likely, however, that a large cohort of undiagnosed patients with a lower severity illness but with digestive symptoms (e.g. diarrhoea, vomiting) may have spread the virus without respiratory involvement [14].

A recent systematic review evaluating a cohort of more than 12,500 patients from 78 studies, found that the pooled prevalence of GI symptoms was $17 \%$; symptoms were evaluated singularly and the weighted prevalence of diarrhea was $12.4 \%$, nausea and/or vomiting $9.0 \%$, loss of appetite $22.3 \%$, and abdominal pain $6.2 \%$. Anyway, the mortality rate among patients with GI symptoms was similar to the overall mortality $[15,16]$.

A meta-analysis by Hayashi et al. on 4 studies compared COVID-19 patients with and without digestive symptoms; they found abdominal pain to be more frequent in patients with a severe form of disease (OR 2.70), suggesting how this kind of symptom could somehow predict a greater degree of severity of disease [17].

Our study aimed at evaluating differences between patients with and without digestive symptoms in terms of personal, clinical and laboratory parameters; moreover, we tried to understand whether digestive symptoms, in general, could somehow be predictive of different outcomes of COVID-19.

\section{Materials and methods}

This is a two single-center retrospective cohort study. We enrolled 495 adult inpatients ( $\geq 18$ years old) who were consecutively hospitalized with SARS-CoV-2 infection, between March and July 2020, in the "Arcispedale S. Anna" in Cona (Fe) and in the "Ospedale del Delta" in Lagosanto (Fe), the two main hospitals serving the Province of Ferrara. The diagnosis of COVID-19 was determined by at least one SARS-CoV-2 RNA detection at oro- and naso-pharyngeal swab and the presence of symptoms clearly related to the infection itself.

The exclusion criteria were: age (subjects younger than 18 years were excluded), the negativity of swabs to viral detection and/or the positivity of stool cultures to the most common gastrointestinal microorganisms.

The study population was divided into two groups: the "case" group, that was constituted by patients with a gastrointestinal onset and symptoms such as nausea, vomiting and/or diarrhea (Digestive Symptoms Group, DSG) and the "control" group, composed by patients who presented without digestive symptoms at the onset of the disease (Non-Digestive Symptoms Group, NDSG). We did not make any distinction among patients in terms of digestive findings, but we calculated a composite gastrointestinal symptoms' prevalence.

Patients' demographic (age, sex), clinical (number of comorbidities) and laboratory data (inflammation and organ damage indexes, samples for viral RNA detection) were extracted from their electronic health records and registered into an electronic database available only to the authors. Data were later anonymized so that no patient could be identified anymore.

We evaluated the load of comorbidities by using the Charlson Comorbidity Index (CCI) which already showed a certain degree of reliability in some studies towards 
mortality by COVID-19 [18, 19]; the items considered by the CCI are shown in Additional file 1: Table S1.

Inflammation was evaluated by using the white blood cells count (WBC), C-reactive protein (CRP), procalcitonin and ferritin levels; the organ damage was appraised, instead, by using serum creatinine, alanine transferase (ALT), isoamylase, lactic dehydrogenase (LDH) and creatine phosphokinase (CPK); the lymphocytes count was also considered.

The aims of this study was four-fold: (1) to determine the prevalence of gastrointestinal presentation among our cohort of patients hospitalized for COVID-19; (2) to evaluate differences between COVID-19 patients with and without presenting digestive symptoms at presentation, (3) to assess differences among males and females COVID-19 inpatients with gastrointestinal symptoms at presentation, and (4) to evaluate whether the presentation with gastrointestinal onset symptoms is associated with better, indifferent or worse outcomes. We chose as COVID outcomes the following: the need for intensification of care (meant as the need for oro-tracheal intubation or non-invasive ventilation), the in-hospital mortality and the 100-day mortality.

The length of stay was defined as the total number of days spent by the subjects in the hospital setting. With "intensification of care" we defined the need for orotracheal intubation or non-invasive ventilation; the observation period was continued until the $100^{\text {th }}$ day since hospital admission, in order to establish the 100-day mortality rate of our cohort of patients.

We followed STROBE (Strenghtening the Reporting of Observational Studies in Epidemiology) guidelines for reporting observational studies as for the compilation of this manuscript.

The local Ethics Committee (Comitato Etico di Area Vasta Emilia Centro, CE-AVEC), belonging to the "Azienda Ospedaliero-Universitaria" in Bologna, "S.Orsola-Malpighi" Hospital, approved the protocol of this study (code:527/2020/Oss/AUSLFe + AOUFe).

\section{Statistical analysis}

The normal distribution of the continuous variables was analysed using Kolmogorov-Smirnov and Shapiro-Wilk tests. Variables not normally distributed were log transformed before entering parametric statistical analysis. Categorical variables were summarized by using frequencies and percentages, while continuous data were presented as median (interquartile range, IQR).

The Mann-Whitney $U$ test was used for continuous variables, and the $x^{2}$ test was used for categorical variables. Variables with a $p$ value $<0.05$ in the univariate analyses were entered into multivariate logistic regression analyses. The Cox regression analyses were performed for evaluating the survival functions of both the groups of patients on statin therapy and not on statin therapy. All $p$ values $<0.05$ are considered statistically significant.

\section{Results}

Between January and July 2020, 495 adult patients were consecutively admitted at our institutions with SARSCoV-2 infection. Of these, 62 (12.5\%) presented with digestive symptoms only (i.e., nausea, vomiting and or diarrhea), while the remaining patients did not have any gastrointestinal manifestation at the onset of COVID-19.

Table 1 illustrates demographic, clinical, and laboratory data at admission of our patients; these were divided into two groups according to the presentation of disease with (DSG) or without (NDSG) gastrointestinal symptoms. DSG patients were significantly younger than those of NDSG group ( $70 \pm 17$ vs. $72 \pm 17$ years, $p=0.043$ ) and with a higher load of comorbidity calculated through their Charlson Comorbidity Index (2 vs. 1 points, $p=0.045)$. The sex distribution was also different between groups, with a greater prevalence of females among DSG patients (62.9\% vs. $48.3 \%, p=0.031)$, while we found no significant difference between groups in terms of laboratory findings.

Comorbidities were evaluated separately (Table 2) and the only significant differences between DSG and NDSG groups concerned the percentage of patients with heart failure (1.6\% vs. $12.7 \%, p=0.009)$ and chronic obstructive pulmonary disease COPD ( $0 \%$ vs. $10.4 \%, p=0.008$ ): anyway, such data cannot be considered consistent because of the small size of the sample involved.

None of the subjects, among our patients, suffered from inflammatory bowel diseases (IBD), neither in the DSG group, nor in the NDSG one; among DSG subjects, 3 had hepatic steatosis in their clinical history $(4.8 \%)$ and 2 an end-stage liver disease (3.2\%). 17 patients of the DSG group (27.4\%) took protonic pump inhibitors (PPI) before hospital admission; moreover, all subjects in the two groups were of Caucasian origin.

DSG patients showed a tendency towards a shorter duration of hospital stay ( $18 \pm 13$ days vs. $20 \pm 15$ days, $p=0.038$ ), while we found no significant differences between DSG and NDSG patients concerning the three outcomes of disease chosen. ((1) The need for intensification of care; (2) The in-hospital death; (3) The 100-day death), as shown in Table 3.

Basing on the different distribution of males and females between groups, we decided to perform new analyses concerning demographic, clinical and laboratory data in the DSG group. For each parameter, we searched for significant differences between males and females (Table 4). 
Table 1 Demographic, clinical, and laboratory findings of patients with COVID-19 on hospital admission, divided for groups (DSG vs. NDSG)

\begin{tabular}{|c|c|c|c|c|}
\hline & DSG $(N=62)$ & NDSG $(\mathrm{N}=433)$ & Overall $(\mathrm{N}=495)$ & $p$ value \\
\hline Sex & & & & 0.031 \\
\hline Male, n (\%) & $23(37.1)$ & $224(51.7)$ & $247(49.9)$ & \\
\hline Female, n (\%) & $39(62.9)$ & $209(48.3)$ & $248(50.1)$ & \\
\hline Age, years $\pm S D$ & $70 \pm 17$ & $72 \pm 17$ & $72 \pm 17$ & 0.042 \\
\hline $\mathrm{CCl}$, points & $2(0-4)$ & $1(0-3)$ & $1(0-3)$ & 0.046 \\
\hline White Blood Cells, n/mmc & 6040 (5130-9450) & $6610(4935-9585)$ & $6540(5020-9670)$ & 0.75 \\
\hline Lymphocytes, n/mmc & $1085(785-1485)$ & $1010(700-1420)$ & $1015(710-1463)$ & 0.24 \\
\hline CRP, mg/dl & $6.6(2.1-13.2)$ & $5.6(1.8-11.6)$ & $5.6(1.9-11.8)$ & 0.31 \\
\hline Procalcitonin, ng/ml & $0.22(0.06-0.43)$ & $0.20(0.07-0.64)$ & $0.20(0.07-0.62)$ & 0.98 \\
\hline Creatinine, mg/dl & $0.94(0.77-1.42)$ & $0.97(0.74-1.28)$ & $0.96(0.75-1.29)$ & 0.60 \\
\hline Isoamylase, U/L & $30(19-54)$ & $32(21-51)$ & $32(21-52)$ & 0.92 \\
\hline $\mathrm{ALT}, \mathrm{U} / \mathrm{L}$ & $19(14-34)$ & $23(14-37)$ & $22(14-36)$ & 0.51 \\
\hline $\mathrm{LDH}, \mathrm{mg} / \mathrm{dl}$ & $282(202-410)$ & $268(199-363)$ & $269(200-368)$ & 0.31 \\
\hline CPK, U/L & $86(48-181)$ & $95(46-179)$ & $89(46-178)$ & 0.73 \\
\hline
\end{tabular}

Data are reported as median (IQR) unless otherwise specified

DGS Digestive Symptoms group, NDGS No Digestive Symptoms Group, SD Standard Deviation, CCI Charlson Comorbidity Index, CRP C Reactive Protein, ALT Alanine Transferase, $L D H$ Lactic Dehydrogenase, CPK Creatine Phosphokinase

Table 2 Comorbidities on admission, divided for groups (DSG vs. NDSG)

\begin{tabular}{llll}
\hline Comorbidity & DSG (N=62) & NDSG (N=433) & $\boldsymbol{p}$ value \\
\hline Hypertension & $41(66.1)$ & $265(61.2)$ & 0.52 \\
Diabetes & $19(30.6)$ & $89(20.6)$ & 0.07 \\
Ischemic Heart Disease & $5(8.1)$ & $58(13.4)$ & 0.23 \\
Heart Failure & $1(1.6)$ & $55(12.7)$ & 0.009 \\
Chronic Kidney Disease (III-IV-V stage) & $8(12.9)$ & $55(12.7)$ & 1.00 \\
Stroke or TIA & $9(14.5)$ & $52(12.0)$ & 0.84 \\
PCOA & $5(8.1)$ & $23(5.3)$ & 0.38 \\
COPD & $0(0)$ & $45(10.4)$ & 0.008 \\
Hepatic Steatosis & $3(4.8)$ & $9(2.1)$ & 0.19 \\
End-stage Liver Disease & $2(3.2)$ & $6(1.4)$ & 0.29 \\
Localized or Hematological Cancer & $15(24.2)$ & $70(16.2)$ & 0.13 \\
Metastatic Cancer & $6(9.7)$ & $21(4.8)$ & 0.12 \\
\hline Allda & & \\
\hline
\end{tabular}

All data are expressed with number of cases (\%)

DGS Digestive Symptoms group, NDGS No Digestive Symptoms Group, TIA Transitory Ischemic Attack, PCOA Peripheral Chronic Obstructive Arteriopathy, COPD Chronic Obstructive Pulmonary Disease

Table 3 Clinical outcomes differences between groups (DSG vs. NDSG)

\begin{tabular}{lllll}
\hline & DSG $(\mathbf{N}=\mathbf{6 2})$ & NDSG $(\mathbf{N}=\mathbf{4 3 3})$ & Overall $(\mathbf{N}=\mathbf{4 9 5 )}$ & $\boldsymbol{p}$ value \\
\hline Length of Stay, days \pm SD & $18 \pm 13$ & $20 \pm 15$ & $19 \pm 15$ & 0.038 \\
Intensification of Care, $\mathrm{n}(\%)$ & $14(22.6)$ & $108(24.9)$ & $122(24.6)$ & 0.69 \\
In-hospital death, $\mathrm{n}(\%)$ & $13(21.0)$ & $108(24.9)$ & $121(24.4)$ & 0.50 \\
100-day Death, $\mathrm{n}(\%)$ & $18(29.0)$ & $132(30.5)$ & $150(30.3)$ & 0.82 \\
\hline
\end{tabular}

Data are reported as number of subjects (\%) unless otherwise specified

DGS Digestive Symptoms group, NDGS No Digestive Symptoms Group, SD Standard Deviation 
Table 4 Demographic, clinical, and laboratory findings in males and females in the Digestive Symptoms Group (DSG)

\begin{tabular}{|c|c|c|c|}
\hline & \multicolumn{2}{|l|}{ DSG $(N=62)$} & \multirow[t]{2}{*}{$p$ value } \\
\hline & Males $(\mathrm{N}=23)$ & Females $(\mathrm{N}=39)$ & \\
\hline Age, years $\pm S D$ & $66 \pm 18$ & $72 \pm 17$ & 0.022 \\
\hline $\mathrm{CCl}$, points & $2(1-5)$ & $1(0-1)$ & 0.034 \\
\hline $\begin{array}{l}\text { White Blood Cells, n/ } \\
\text { mmc }\end{array}$ & 6040 (5130-9650) & $6140(5640-9140)$ & 0.60 \\
\hline Lymphocytes, n/mmc & 985 (380-1000) & 1155 (1180-1890) & 0.38 \\
\hline CRP, mg/dl & $11.1(9.4-21.3)$ & $4.9(2.0-8.0)$ & 0.043 \\
\hline Procalcitonin, ng/ml & $0.28(0.10-5.83)$ & $0.18(0.02-0.43)$ & 0.14 \\
\hline Creatinine, mg/dl & $1.09(0.79-1.46)$ & $0.86(0.75-1.24)$ & 0.22 \\
\hline Isoamylase, U/L & $32(19-46)$ & $30(23-33)$ & 0.99 \\
\hline $\mathrm{ALT}, \mathrm{U} / \mathrm{L}$ & $22(15-42)$ & $18(13-23)$ & 0.25 \\
\hline $\mathrm{LDH}, \mathrm{mg} / \mathrm{dl}$ & $369(209-425)$ & $246(183-296)$ & 0.07 \\
\hline CPK, U/L & $88(63-187)$ & $63(86-134)$ & 0.34 \\
\hline
\end{tabular}

Data are reported as median (IQR) unless otherwise specified

DGS Digestive Symptoms group, NDGS No Digestive Symptoms Group, SD Standard Deviation; CRP, C Reactive Protein, ALT Alanine Transferase, LDH Lactic Dehydrogenase, CPK Creatine Phosphokinase

Females, in particular, tended to a shorter length of stay $(17 \pm 13$ vs. $20 \pm 14$ days, $p=0.011)$, while there were not substantial differences between sexes in terms of need for intensification of care, in-hospital death and death after 100 days. These findings are reported in Table 5.

Logistic regression analyses were also performed choosing those variables that confirmed to be statistically different between groups; we tried to understand which of the factors considered in our work could somehow independently determine a better or a worse prognosis in our cohort of patients. Anyway, none of the variables tested resulted to be independently associated with COVID-19 outcomes (data not shown).

To understand the role of the variables sex, age and CCI, supposing them to be the main selection bias of our findings, we decided to perform two separate Cox regression analyses, that determined the survival curves of the population with and without gastrointestinal onset (DSG and NDSG, respectively). The Cox regression analysis in Fig. 1, tab. a, shows the survival curves of both populations after 100 days of observation without any adjustment. In tab. b of Fig. 1 it is shown the Cox regression analysis for the same populations of patients but with the adjustment for sex, age and comorbidities (weighted through the $\mathrm{CCI}$ ): the population of patients with digestive symptoms only at the debut of COVID-19 seems to

Table 5 Clinical outcomes measure of patients in the Digestive Symptoms Group (DSG): Males vs. Females

\begin{tabular}{lllll}
\hline & Males $(\mathbf{N}=\mathbf{2 3})$ & Females $(\mathbf{N}=\mathbf{3 9})$ & Overall $(\mathbf{N}=\mathbf{6 2})$ & $\boldsymbol{p}$ value \\
\hline Length of Stay, days \pm SD & $20 \pm 14$ & $17 \pm 13$ & $18 \pm 13$ & 0.011 \\
Intensification of Care, $\mathrm{n}(\%)$ & $7(30.4)$ & $7(17.9)$ & $14(22.6)$ & 0.26 \\
In-hospital death, $\mathrm{n}(\%)$ & $5(21.7)$ & $8(20.5)$ & $13(21.0)$ & 0.91 \\
100-day Death, $\mathrm{n}(\%)$ & $6(26.1)$ & $12(30.8)$ & $18(29.0)$ & 0.70 \\
\hline
\end{tabular}

Data are reported as number of subjects (\%) unless otherwise specified

DGS Digestive Symptoms group, NDGS No Digestive Symptoms Group, SD Standard Deviation

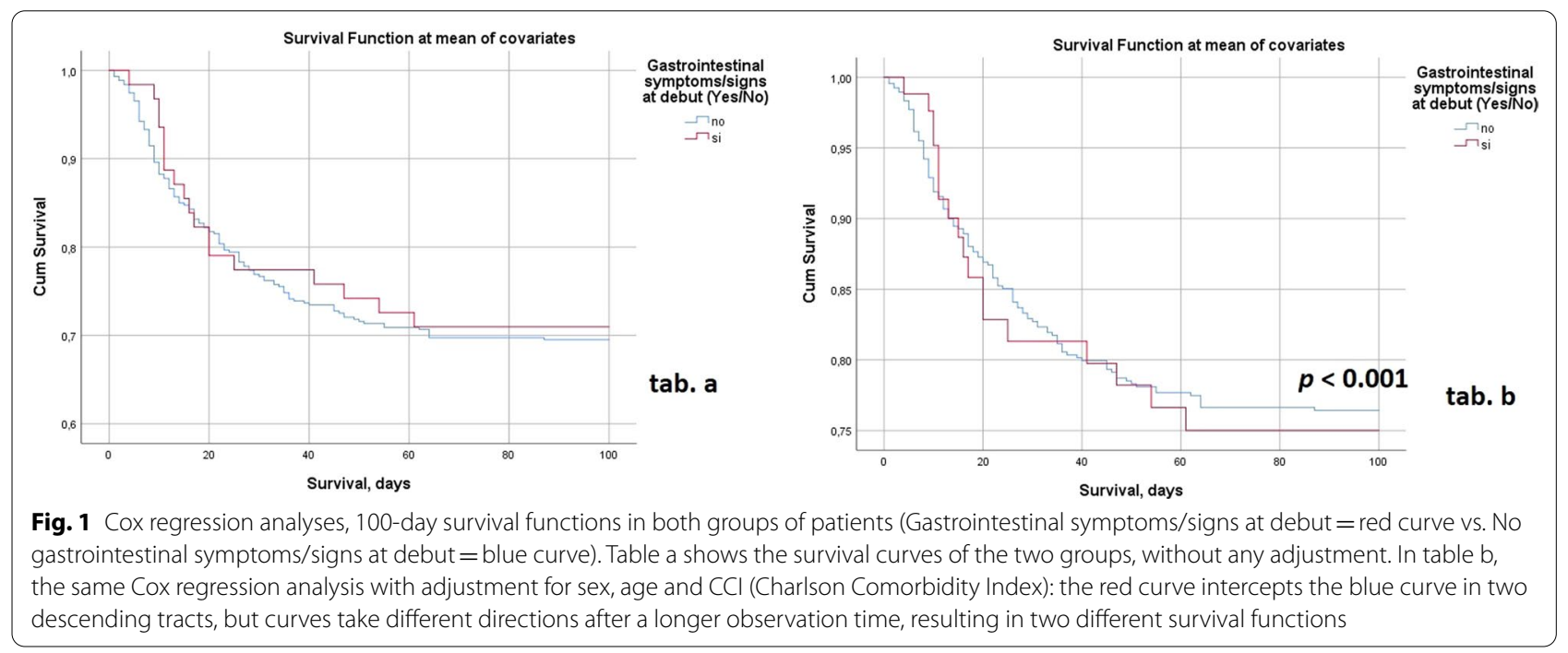


survive longer if compared to that of patients without gastrointestinal onset $(p<0.001)$.

\section{Discussion}

In this study, we enrolled 495 patients, consecutively hospitalized to our two hospitals of Ferrara's territory dedicated to COVID-19 inpatients. Among the patients hospitalized for SARS-CoV-2 infection, the prevalence of gastrointestinal symptoms at presentation was $12.5 \%$. COVID-19 patients presenting with digestive symptoms only, as opposed to those without, showed a prevalence of females, and these ones tended to a shorter hospital stay.

One of the first case series published in China on 40 COVID-19 inpatients reported that the main symptom was dyspnoea (55\%) while diarrhoea was described in 3\% of cases only; the median age of subjects was 49 years [1]. Other Chinese studies reported different percentage of gastrointestinal symptoms [20, 21]: Zhang et al. reported fever (91\%), cough (75\%), fatigue (75\%) and gastrointestinal symptoms (39\%) as the most common clinical manifestations in 140 hospitalized COVID -19 patients while Wang et al. found a gastrointestinal onset rate of $10.1 \%$ in a group of 138 hospitalized patients with a median age of 56 years. Cheung et al., in their meta-analysis of 60 studies involving 4,243 COVID-19 patients from six countries, found digestive symptoms in $17.6 \%$ of the patients with specific rates of anorexia (26.8\%), diarrhoea (12.5\%), nausea/vomiting (10.2\%) and abdominal pain/discomfort (9.2\%). In this meta-analysis, authors suggested that gastrointestinal symptoms are more common in the severe form of the disease (17.1\%) [22].

In a meta-analysis on 118 international subjects by Sultan et al., evaluating also subjects outside of China, gastrointestinal symptoms were reported in $10 \%$ of cases, while ALT increases were found in $15-20 \%$ of the subjects involved in the analysis. Diarrhea was present in $6.2 \%$ of all inpatients from China $(8.9 \%$ in the overall analyses, regardless of hospitalization), while as for non-Chinese patients these percentage was much higher (18.9\% among the inpatients, $20.1 \%$ in the overall population); analyses were performed also considering diarrhea as presenting symptoms of the disease with a percentage of $9.3 \%$ in the overall population $(8.0 \%$ among Chinese subjects and $20.0 \%$ in the non-Chinese cohort). Nausea and/or vomiting were reported in $7.6 \%$ of the overall population, regardless of hospitalization (4.9\% of Chinese subjects, $15.9 \%$ among non-Chinese subjects); abdominal pain was, instead, present in $4.0 \%$ of patients (3.0\% among Chinese subjects, $6.4 \%$ among non-Chinese subjects) [23]. Such data could suggest a sort of regional difference in the predisposition to digestive symptoms, according to which the Chinese population would manifest COVID-19 in a lower percentage of cases with gastrointestinal onset. Studies concerning this aspect are still missing and it is still impossible to make any kind of conclusion about it. Some studies were also performed in the pediatric population and a very recent meta-analysis by Bolia et al. [24] evaluated the prevalence rates of diarrhea, nausea/vomiting and abdominal pain among young subjects: such symptoms were reported to be present in nearly $20 \%$ of patients and were associated with the severe clinical form of COVID-19.

In order to compare the outcomes of patients in both DSG and NDSG groups, we evaluated the duration of length of hospital stay, the need for intensification of care, the in-hospital death and the global 100-day death. COVID-19 patients presenting with digestive symptoms tended to have a shorter hospital stay, but showed no difference concerning the major outcomes of the disease, differently from the studies cited above. In addition, in the DSG there was a prevalence of females $(62.9 \%$ vs. 48.3\%) and, for this reason, we decided to extend our analyses to search for differences between males and females in terms of age, comorbidities (through the CCI score), inflammation and organ damage indexes, finding males to be younger than females (median age $66 \pm 18$ vs. $72 \pm 17$ years), on average more comorbid and with higher C-reactive protein levels. No differences among sexes were found in terms of COVID-19 outcomes, even if female inpatients tended to a shorter length of hospital stay.

The debut of disease with gastrointestinal symptoms was initially associated with a better prognosis of COVID-19, and usually intended as a mild or moderate illness $[6,8]$. The opposite was observed in the population with a respiratory presentation of the disease, which could go towards mild, moderate or severe diseases depending on the case [13].

Further analyses evaluating a greater number of subjects showed completely different associations, as already stated above, with digestive symptoms more often associated to worse outcomes, in general. Many hypotheses were made about this unfavourable association by the gastrointestinal onset; the most accredited hypothesis states that the debut of the disease at a digestive level would somehow delay the hospital admission, indirectly allowing a greater viral intracellular replication, leading to a late treatment and, thereby, a worse prognosis. However, whether intestinal lesions of COVID-19 are the result of a secondary response after systemic inflammation, are the result of a primary intestinal infection, or are the combined results of both mechanisms remains uncertain.

Another interesting point of view concerns the sex differences found in this study. Previous works showed 
that females, due to higher innate cellular and humoral immune responses, react differently than males to both viral infections and vaccines [25]. Other authors supported the idea of different sex-specific responses to viral infections during acute virological control and in immunopathologic manifestations [26].

It is possible that female sex gives some kind of immune protection against SARS-CoV-2, as it happens for other viruses, through complex interactions between sex hormones, sex chromosomes, and immune response genes. This could lead to a different presentation of disease in males that could partially explain the difference in prevalence among females and males in our population with gastrointestinal presentation.

The survival functions of DSG and NDSG groups showed that the patients with gastrointestinal onset have a more favourable prognosis of the disease after 100 days. This could have been partially explained by the sort of "protection" that the female sex gives towards SARS-CoV-2 infection. For this reason, we decided to adjust the COX regression analysis for sex, as well as for age and comorbidities, with significant results, particularly in contrast with some of the findings previously cited.

It is still unclear why the gastrointestinal colonization by SARS-CoV-2 effectively affects the prognosis of patients, nor how this could happen. Thus, despite studies about this specific aspect of disease are day by day increasing, it is necessary to further investigate the clinical aspects of COVID-19 patients presenting with gastrointestinal symptoms.

This study has several limitations, and our findings must be read with caution. The main limitations of this work are attributable, in the first instance, to its retrospective nature and the inclusion of a limited number of COVID-19 patients presenting with digestive symptoms. Moreover, it was not possible to evaluate singularly all the possible etiologic factors underlying the gastrointestinal onset of the disease: the small size of sample, in fact, did not allow to perform specific analyses for each of them.

\section{Conclusions}

The prevalence of digestive symptoms at the debut of COVID-19 in our cohort of inpatients was $12.5 \%$. In the group of patients with gastrointestinal onset (DSG) there was a greater prevalence of females and subjects were significantly younger but with a higher load of comorbidities (this was expressed through the evaluation of the Charlson Comorbidity Score, CCI). Exploring the differences between sexes among DSG patients, we found that males were younger than females, with a greater CCI score, and with higher serum C-reactive protein; moreover, females tended to a shorter length of stay than males, while there was no difference in terms of prevalence concerning the major outcomes of the disease.

COX regression analyses showed more favourable survival functions after 100 days by the DSG patients, if adjusting the test for age, sex and comorbidities.

\section{Supplementary Information}

The online version contains supplementary material available at https://doi. org/10.1186/s12879-021-06476-y.

Additional file 1: Table S1. The components of the Charlson Comorbidity Index.

\section{Acknowledgements}

We would like to dedicate our sincere thanks to all the members of the medical staff that is still participating in the fight against this pandemic, to all the extremely skillful and valuable nursing staff members as well as our assistance personnel, every day working on the frontline. Special thanks to the directors of the Internal Medicine Units where our research took place: Giovanni Zuliani MD, PhD, director of the "University Internal Medicine" department of "Arcispedale S.Anna" in Cona (Fe), Roberto Manfredini MD, PhD, director of the "Clinical Medicine" department of "Arcispedale S.Anna" in Cona (Fe) and Stefano Parini, MD, director of the "Internal Medicine" department of "Ospedale del Delta" in Lagosanto (Fe).

\section{Authors' contributions}

SG and NF: acquisition, analysis and interpretation of data; drafting the article. $A B$ and BB: acquisition, analysis and interpretation of data. SP, SR, SG and MG: acquisition of data. AP: conception and design of the study; drafting the article and revising it critically for important intellectual content, final approval of the version to be submitted. CVF: drafting the article and revising it critically for important intellectual content, final approval of the version to be submitted. All authors revised the article critically for important intellectual content and approved its final version for submission. All authors read and approved the final manuscript.

Funding

No funding was provided to support the preparation of this article.

Availability of data and materials

Data are protected by a research consortium. The Corresponding Author will share the data upon formal proposal of the interested researchers.

\section{Declarations}

\section{Ethics approval and consent to participate}

The local Ethics Committee (Comitato Etico di Area Vasta Emilia Centro, CE-AVEC) approved the protocol of this study (code:527/2020/Oss/AUS$\mathrm{LFe}+\mathrm{AOUFe}$ ). All participants provided written informed consent for participation to the study.

\section{Consent to publish}

Not applicable.

\section{Competing interests}

On behalf of all authors, the corresponding author states that there is no conflict of interest. The authors declare that they have no known competing financial interests or personal relationships that could have appeared to influence the work reported in this paper.

\section{Author details}

${ }^{1}$ Department of Translational Medicine, University of Ferrara, Ferrara, Italy - Via Luigi Borsari, 46, 44121 Ferrara, Emilia Romagna, Italy. ${ }^{2}$ Department of General Surgery, Azienda Unità Sanitaria Locale Di Ferrara, Ospedale del Delta, via Valle 
Oppio, 2, Lagosanto, 44023 Ferrara, Emilia Romagna, Italy. ${ }^{3}$ Medical Department, Azienda Unità Sanitaria Locale Di Ferrara, Ospedale del Delta, via Valle Oppio, 2, Lagosanto, 44023 Ferrara, Emilia Romagna, Italy. ${ }^{4}$ Medical Department, University Hospital of Ferrara Arcispedale Sant'Anna, Via Aldo Moro, 8, 44124 Ferrara, Emilia Romagna, Italy. ${ }^{5}$ Department of Translational Medicine and Medical Department, University of Ferrara, University Hospital of Ferrara Arcispedale Sant'Anna, Via Aldo Moro, 8, 44124 Ferrara, Emilia Romagna, Italy.

Received: 29 October 2020 Accepted: 27 July 2021

Published online: 03 August 2021

\section{References}

1. Huang C, Wang Y, Li X, Ren L, Zhao J, Hu Y, et al. Clinical features of patients infected with 2019 novel coronavirus in Wuhan, China. Lancet. 2020;395(10223):497-506.

2. Zhang W, Zhao Y, Zhang F, Wang Q, Li T, Liu Z, et al. The use of anti-inflammatory drugs in the treatment of people with severe coronavirus disease 2019 (COVID-19): The experience of clinical immunologists from China. Vol. 214, Clinical Immunology. Academic Press Inc.; 2020. p. 108393.

3. Channappanavar R, Perlman S. Pathogenic human coronavirus infections: causes and consequences of cytokine storm and immunopathology. Vol. 39, Seminars in Immunopathology. Springer Verlag; 2017. p. 529-39.

4. Perisetti A, Goyal H, Gajendran M, Boregowda U, Mann R, Sharma N. Prevalence, Mechanisms, and Implications of Gastrointestinal Symptoms in COVID-19. Vol. 7, Frontiers in Medicine. Frontiers Media S.A.; 2020 [cited 2021 Jun 5]. p. 588711. www.frontiersin.org.

5. Wu Z, McGoogan JM. Characteristics of and Important Lessons from the Coronavirus Disease 2019 (COVID-19) Outbreak in China: Summary of a Report of 72314 Cases from the Chinese Center for Disease Control and Prevention. JAMA. 2020;323(13):1239-42.

6. Lin L, Jiang X, Zhang Z, Huang S, Zhang Z, Fang Z, et al. Gastrointestinal symptoms of 95 cases with SARS-CoV-2 infection. Gut. 2020:69(6):997-1001.

7. Zhou F, Yu T, Du R, Fan G, Liu Y, Liu Z, et al. Clinical course and risk factors for mortality of adult inpatients with COVID-19 in Wuhan, China: a retrospective cohort study. Lancet. 2020;395(10229):1054-62.

8. Xiao F, Tang M, Zheng X, Liu Y, Li X, Shan H. Evidence for Gastrointestinal Infection of SARS-CoV-2. Gastroenterology. 2020;158(6):1831-3.

9. Chan JFW, Yuan S, Kok KH, To KKW, Chu H, Yang J, et al. A familial cluster of pneumonia associated with the 2019 novel coronavirus indicating person-to-person transmission: a study of a family cluster. Lancet. 2020;395(10223):514-23.

10. Zhou P, Yang X-L, Wang X-G, Hu B, Zhang L, Zhang W, et al. A pneumonia outbreak associated with a new coronavirus of probable bat origin. Nature. 2020;579(7798):270-3.

11. Hikmet F, Méar L, Edvinsson $\AA$, Micke $P$, Uhlén $M$, Lindskog C. The protein expression profile of ACE2 in human tissues. Mol Syst Biol. 2020;16:7.

12. Fagerberg L, Hallström BM, Oksvold P, Kampf C, Djureinovic D, Odeberg J, et al. Analysis of the human tissue-specific expression by genome-wide integration of transcriptomics and antibody-based proteomics. Mol Cell Proteomics. 2014;13(2):397-406.

13. Duca A, Memaj I, Zanardi F, Preti C, Alesi A, Della Bella L, et al. Severity of respiratory failure and outcome of patients needing a ventilatory support in the emergency department during italian novel coronavirus
SARS-CoV-2 outbreak: preliminary data on the role of helmet CPAP and non-invasive ventilation. SSRN Electron J. 2020;24:100419.

14. Cha MH, Regueiro M, Sandhu DS. Gastrointestinal and hepatic manifestations of COVID-19: a comprehensive review. World J Gastroenterol. 2020:26(19):2323-31.

15. Pareek M, Singh A, Vadlamani L, Eder M, Pacor J, Park J, et al. Relation of cardiovascular risk factors to mortality and cardiovascular events in hospitalized patients with coronavirus disease 2019 (From the Yale COVID-19 Cardiovascular Registry). Am J Cardiol. 2019. https://doi.org/10.1016/j. amjcard.2021.01.029.

16. Tariq R, Saha S, Furqan F, Hassett L, Pardi D, Khanna S. Prevalence and Mortality of COVID-19 patients with gastrointestinal symptoms: a systematic review and meta-analysis. Mayo Clin Proc. 2020;95(8):1632-48.

17. Hayashi Y, Wagatsuma K, Nojima M, Yamakawa T, Ichimiya T, Yokoyama $Y$, et al. The characteristics of gastrointestinal symptoms in patients with severe COVID-19: a systematic review and meta-analysis. J Gastroenterol. 2021;56:409-20.

18. Charlson ME, Pompei P, Ales KL, MacKenzie CR. A new method of classifying prognostic comorbidity in longitudinal studies: development and validation. J Chronic Dis. 1987:40(5):373-83.

19. Quan H, Li B, Couris CM, Fushimi K, Graham P, Hider P, et al. Updating and validating the Charlson comorbidity index and score for risk adjustment in hospital discharge abstracts using data from 6 countries. Am J Epidemiol. 2011;173(6):676-82

20. Zhang J, Dong X, Cao Y, Yuan Y, Yang Y, Yan Y, et al. Clinical characteristics of 140 patients infected with SARS-CoV-2 in Wuhan. China Allergy. 2020. https://doi.org/10.1111/all.14238.

21. Wang D, Hu B, Hu C, Zhu F, Liu X, Zhang J, et al. Clinical Characteristics of 138 Hospitalized Patients With 2019 Novel Coronavirus-Infected Pneumonia in Wuhan. China JAMA. 2020;323(11):1061-9.

22. Cheung KS, Hung IFN, Chan PPY, Lung KC, Tso E, Liu R, et al. Gastrointestinal Manifestations of SARS-CoV-2 Infection and Virus Load in Fecal Samples From a Hong Kong Cohort: Systematic Review and Meta-analysis. Gastroenterology. 2020;159(1):81-95.

23. Sultan S, Altayar O, Siddique SM, Davitkov P, Feuerstein JD, Lim JK, et al. AGA Institute Rapid Review of the Gastrointestinal and Liver Manifestations of COVID-19, Meta-Analysis of International Data, and Recommendations for the Consultative Management of Patients with COVID-19. Gastroenterology. 2020;159(1):320-34

24. Bolia R, Dhanesh Goel A, Badkur M, Jain V. Gastrointestinal manifestations of pediatric coronavirus disease and their relationship with a severe clinical course: a systematic review and meta-analysis. J Trop Pediatr. 2021. https://doi.org/10.1093/tropej/fmab051/6288463.

25. Ruggierii A, Anticoli S, D'ambrosio A, Giordani L, Mora M. The influence of sex and gender on immunity, infection and vaccination. Ann Ist Super Sanita. 2016:52(2):198-204.

26. Ghosh S, Klein RS. Sex Drives Dimorphic Immune Responses to Viral Infections. J Immunol. 2017;198(5):1782-90. http://www.jimmunol.org/ content/198/5/1782. http://www.jimmunol.org/content/198/5/1782.full\# ref-list-1.

\section{Publisher's Note}

Springer Nature remains neutral with regard to jurisdictional claims in published maps and institutional affiliations. 